\title{
First detection of photospheric depletion in the Large Magellanic Cloud ${ }^{\star}$
}

\author{
M. Reyniers ${ }^{\star \star}$ and H. Van Winckel \\ Instituut voor Sterrenkunde, Departement Natuurkunde en Sterrenkunde, KU Leuven, Celestijnenlaan 200D, 3001 Leuven, Belgium \\ e-mail: maarten@ster. kuleuven. be
}

Received 5 December 2006 / Accepted 31 December 2006

\section{ABSTRACT}

\begin{abstract}
Context. Recent photospheric abundance studies of galactic field RV Tauri stars show that depletion of refractory elements is rather common in these evolved objects.

Aims. The process that creates this chemical anomaly is not understood well, but it probably requires the presence of gravitationally bound dust in a binary system. We test for the presence of depletion in extra-galactic objects.

Methods. A detailed photospheric abundance study on the basis of high-quality UVES spectra was performed on the RV Tauri star in the LMC: MACHO 82.8405.15. Abundances were derived using a critically compiled line list with accurate $\log (g f)$ values and the latest Kurucz model atmospheres.

Results. With $[\mathrm{Fe} / \mathrm{H}]=-2.6$ in combination with $[\mathrm{Zn} / \mathrm{Fe}]=+2.3$ and $[\mathrm{S} / \mathrm{Ti}]=+2.5, \mathrm{MACHO} 82.8405 .15$ displays a strong depletion abundance pattern. The effect of the depletion is comparable to the strongest depletions seen in field Galactic RV Tauri stars. Conclusions. The chemical analysis of MACHO 82.8405.15 proves that the depletion process also occurs in the extragalactic members of the RV Tauri pulsation class. Our program star is a member of a larger sample of LMC RV Tauri objects. This sample is unique, since the distances of the members are well-constrained. Further studies of this sample are therefore expected to gain deeper insight into the poorly understood depletion phenomenon and of the evolutionary status of RV Tauri stars in general.
\end{abstract}

Key words. stars: AGB and post-AGB - stars: abundances - stars: individual: MACHO 82.8405.15 - Magellanic Clouds

\section{Introduction}

It is now almost one century ago that Seares \& Haynes (1908) noted how RV Tauri, the prototype of the RV Tauri variables, shows a light curve with subsequent deep and shallow minima. This observation, together with the required limits on the spectral type ( $\mathrm{F}$ to $\mathrm{K})$, is still used as the defining characteristic for the RV Tauri variables. Preston et al. (1963) defined three spectroscopic classes: The stars of type RVA have spectral type G-K, and show strong absorption lines but normal $\mathrm{CN}$ or $\mathrm{CH}$ bands. The RVB stars are generally somewhat hotter (spectral type F), weak-lined objects that show enhanced $\mathrm{CN}$ and $\mathrm{CH}$ bands. RVC stars are also weak-lined stars, but they show normal bands of $\mathrm{CH}$ and $\mathrm{CN}$.

The exact evolutionary stage of RV Tauri stars is still not very clear, but they are probably in the post-AGB stage of evolution, as first argued by Jura (1986). Many of the RV Tauri stars show an infrared excess, which is caused by the circumstellar dust that was likely formed by heavy mass loss during the preceding AGB phase. The MACHO experiment in the 90 s revealed the first extragalactic RV Tauri stars in the Large Magellanic Cloud. Alcock et al. (1998) showed the light curves of about a dozen RV Tauri stars in the LMC, with "formal periods" (the time between two successive minima) between 40 and 120 days, defined a single PL relation for these stars, and strengthened the post-AGB interpretation of RV Tauri stars by their luminosities.

* Based on observations collected at the European Southern Observatory, Chile (programme 074.D-0619(A)).

$\star \star$ Postdoctoral fellow of the Fund for Scientific Research, Flanders.
The extensive chemical studies of the photospheres of field RV Tauri stars showed, however, that none of the RV Tauri stars shows evidence of post 3rd dredge-up chemical enrichments, such as enhanced carbon or enhanced s-process abundances (except for maybe V453 Oph, see Deroo et al. 2005). Instead, the abundance studies (see e.g. Giridhar et al. 2000, 2005; Maas et al. 2005, and references therein) reveal that severe abundance anomalies are observed in mainly RVB stars, with underabundances of elements with a high condensation temperature. The origin of these depletion patterns probably involves chemical fractionation due to dust formation in the circumstellar environment, followed by a decoupling of the gas and dust with a reaccretion of the cleaned gas on the stellar photosphere. It was realised that the spectroscopic classes of Preston et al. could be attributed to a different degree of depletion, with the RVB stars the most heavily depleted. The whole process probably requires a stable circumstellar environment, like a stable disk (Waters et al. 1992). Since a stable disk is only likely to form in a binary system, it is believed that all depleted RV Tau stars are probably binaries (Van Winckel 2003; De Ruyter et al. 2006). Note that depletion does not seem to occur in globular cluster RV Tauri stars, or in field RV Tauri stars of low metallicity $([\mathrm{Fe} / \mathrm{H}]<-1)$. Recently, we (Reyniers et al. 2006) reported on the intrinsic metal-poor RV Tauri star MACHO 47.2496.8 in the $\mathrm{LMC}$, which is unique for displaying a very strong s-process enrichment. This corroborates the post-AGB nature of this object, but adds to the chemical diversity observed in this pulsation class.

Preliminary work by Lloyd Evans \& Pollard (2004) showed, on the basis of low-resolution spectra, that at least two RV Tauri stars found through the MACHO experiment are RVB 
Table 1. Log of the high-resolution VLT-UVES observations, with small spectral gaps between $5757 \AA$ and $5833 \AA$ and between $8521 \AA$ and $8660 \AA$ due to the spatial gap between the two UVES CCDs.

\begin{tabular}{|c|c|c|c|c|}
\hline date & $\begin{array}{l}\text { UT } \\
\text { start }\end{array}$ & $\begin{array}{r}\text { exp.time } \\
\text { (s) }\end{array}$ & $\begin{array}{l}\text { wavelength } \\
\text { interval }(\AA)\end{array}$ & $S / N$ \\
\hline $2005-02-08$ & $01: 43$ & 3600 & $4780-6808$ & 130 \\
\hline 2005-02-09 & $02: 37$ & 7200 & $\left\{\begin{array}{r}3758-4983 \\
6705-10084\end{array}\right.$ & $\begin{array}{l}110 \\
120\end{array}$ \\
\hline
\end{tabular}

stars, and hence potentially depleted. In this letter, we focus on the brightest of both objects: MACHO 82.8405.15 $\left(m_{V}=14.3\right)$. Alcock et al. (1998) found a period of 93.1 days for this object and a mean absolute visual magnitude of $M_{V}=-4.52$. The amplitude of the pulsation is significant, with a peak-to-peak of 0.89 in the MACHO $V$ mag. Here, we present a detailed abundance analysis of MACHO 82.8405.15, based on high-resolution, high signal-to-noise VLT-UVES spectra.

\section{Observations and analysis}

Spectra were taken with UVES on the VLT-UT2 (Kueyen) in visitor mode by one of us (HVW). Details of the observations, together with some indicative signal-to-noise $(\mathrm{S} / \mathrm{N})$ ratios, can be found in Table 1. The spectra were taken just before minimum light. The reduction was performed in the dedicated "UVES context" of the ESO MIDAS software packet, including all standard reduction steps. Optimal extraction was used, except for the spectrum taken in the first night, since this was taken with the slicer and optimal extraction is not implemented for this type of data. A sample spectrum can be found in Fig. 1.

The method of analysis is similar to the method used in our previous publications on similar stars (e.g. Deroo et al. 2005). First, a complete line identification was performed, using the line lists of Thévenin $(1989,1990)$. Then, lines with a clean profile and an equivalent width smaller than $150 \mathrm{~m} \AA$ were selected for abundance calculation purposes. The $\log (g f)$ values of the selected lines were taken from the line list with accurate oscillator strengths described in Van Winckel \& Reyniers (2000). Lines without an accurate $\log (g f)$ value were discarded. For the abundance calculation, we used the latest Kurucz models (http: //kurucz . harvard. edu), in combination with the LTE line analysis program MOOG by C. Sneden (April 2002 version). Since initially only $24 \mathrm{Fe}$ I and $3 \mathrm{Fe}$ II lines were found with our usual line list, $11 \mathrm{Fe}$ I and $4 \mathrm{Fe}$ II lines were added from Thévenin $(1989,1990)$, although these papers are generally regarded as a less accurate source for oscillator strengths.

In our search for the atmospheric parameters of MACHO 82.8405.15, we realised that the classical "spectroscopic" method of studying the iron lines failed. The usual technique of demanding excitation equilibrium for the Fe I lines resulted in an effective temperature of $T_{\text {eff }}=7000 \mathrm{~K}$, but such a high effective temperature gives unrealistic supersolar zinc ( $\mathrm{Zn}$ ) abundances. Moreover, at $T_{\text {eff }}=7000 \mathrm{~K}$, ionisation equilibrium can only be attained with a high gravity of $\log g=2.5$. The reason the classical method fails is not clear, but is probably related to the dynamical character of the photosphere (see Sect. 3) and/or to the well-known non-LTE effect of UV overionisation in the atmospheres of late-type stars (e.g. Shchukina et al. 2005). This effect is expected to be greater in metal-poor stars as a result of the lower electron density and of the weaker UV blanketing.
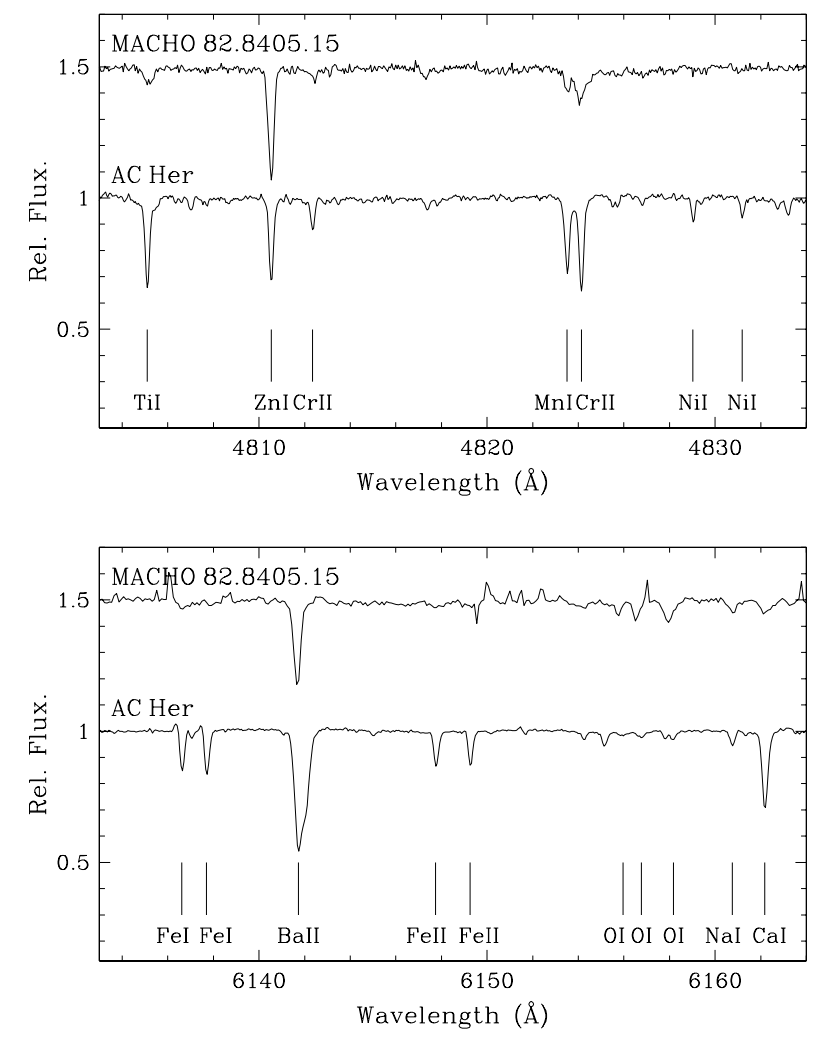

Fig. 1. The VLT+UVES spectrum of MACHO 82.8405.15, together with the spectrum of ACHer, around the $4810 \AA \mathrm{Zn}$ line (upper panel) and the $6155 \AA$ oxygen triplet (lower panel). ACHer is a depleted field RV Tauri star that is somewhat cooler $\left(T_{\text {eff }}=5500 \mathrm{~K}\right)$ than MACHO 82.8405.15. It is extensively discussed in Van Winckel et al. (1998). In the upper panel, the $\mathrm{Zn}$ line is remarkably strong, while lines of other iron peak elements are almost absent. In the lower panel, the oxygen triplet around $6155 \AA$ is clearly visible, indicating the hotter temperature for MACHO 82.8405.15. Note that the spectrum in the lower panel has quite a lot of telluric emission. Correction for this emission is difficult, since this part of the spectrum is observed with the image slicer, hence no sky window is available.

We had to follow an alternative scheme to deduce the atmospheric parameters. First, we estimated the gravity from the luminosity that is given in Alcock et al. (1998). This estimate is of course dependent on the adopted effective temperature, the initial mass, and the reddening. However, for all temperatures compatible with an F-star spectrum, a low gravity of $\log g \leq 1.0$ is found. A further decrease in the parameter space was obtained through fitting the $\mathrm{H} \gamma$ and $\mathrm{H} \delta$ Balmer lines using Kurucz Balmer profiles. The $\mathrm{H} \alpha$ and $\mathrm{H} \beta$ lines were not used for this purpose since they show strong emission features. The wings and the cores of the two lines were fitted simultaneously, with more emphasis on a good fit for the wings. We could constrain the parameter space further to models with $T_{\text {eff }} \leq 6000 \mathrm{~K}$ by this Balmerprofile fitting. From a comparison of photometry taken with the Swiss Euler telescope at La Silla (see Reyniers et al. 2006, for more details on these observations) with synthetic colours, we inferred that the reddening is certainly small, with an upper limit of $E(B-V)=0.2$. If we combine this information with the gravity from the luminosity and the Balmer fits, we could conclude that the only consistent temperature is $T_{\text {eff }}=6000 \mathrm{~K}$. The final choice between the two consistent gravities at this temperature, 0.5 and 1.0, was made on the basis of the consistency in the abundances, resulting in $\log g=0.5$. The $\mathrm{H} \gamma$ and $\mathrm{H} \delta$ Balmer fits 


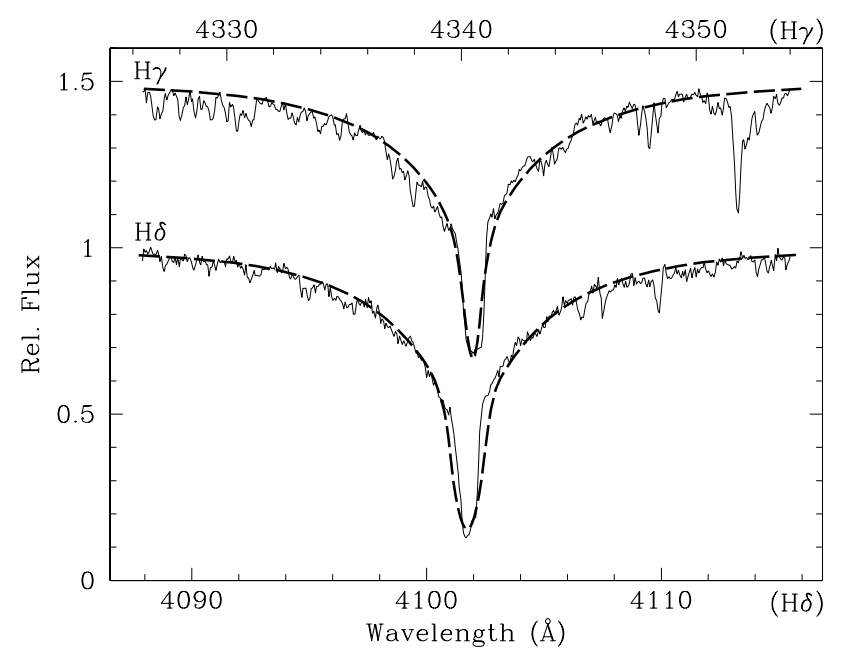

Fig. 2. The final $\mathrm{H} \gamma$ and $\mathrm{H} \delta$ Balmer fits.

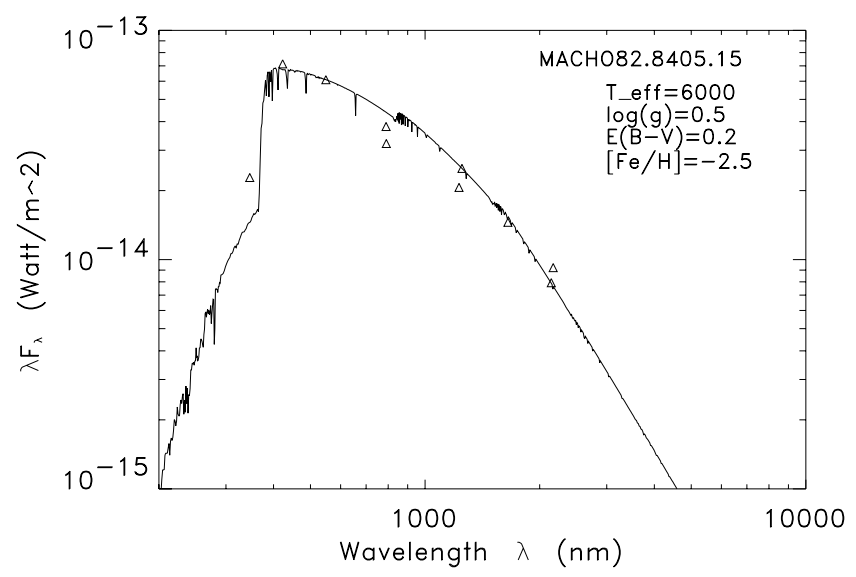

Fig. 3. The spectral energy distribution (SED) of MACHO 82.8405.15. The dereddened photometry is shown with triangles, while the Kurucz model is overplotted with a solid line. The photometry includes: $U$, $B, V$ mag in the Geneva system; $I$ in the Cousins system; $I, J$, and $K$ from DENIS; and $J, H$, and $K$ from 2MASS. The optical photometry shown here is taken at a more luminous phase than the UVES spectroscopy on which the atmospheric parameters are based. The SED fits, however, were only used to constrain the reddening towards MACHO 82.8405.15.

with the final parameters are shown in Fig. 2; the spectral energy distribution is shown in Fig. 3. Note that there is a phase difference of 0.24 between the UVES spectra on which the Balmer fitting was performed and the photometry, implying a high uncertainty in the derived parameters (at least $\pm 250 \mathrm{~K}$ in $T_{\text {eff }}$ and \pm 1.0 in $\log g$ ). We want to stress, however, that in the parameter determination, the photometry was used solely to constrain the reddening towards MACHO 82.8405.15.

\section{Radial velocities of individual lines}

Before turning to the actual abundances, the radial velocities of the individual spectral lines need to be noted. During our analysis, we noticed that some lines were more velocity-shifted than others. Moreover, a clear correlation exists between the radial velocity and the excitation potential of a line, with lower velocities for lines with a high excitation potential. This is illustrated in Fig. 4. It is indicative of an optical depth effect due to a differential velocity field in the line-forming region of the dynamical photosphere of this pulsating star. In field RV Tauri

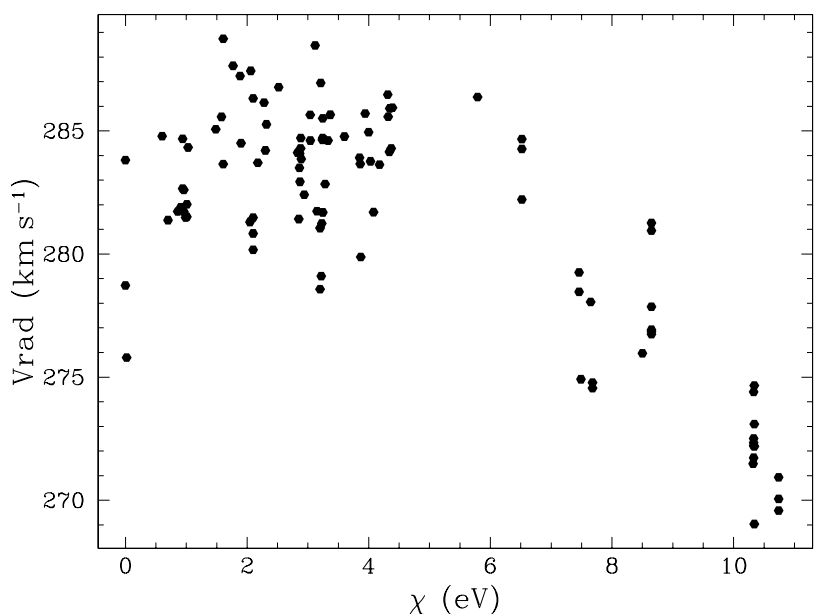

Fig. 4. The clear correlation between the heliocentric radial velocities of individual lines and their lower excitation potentials.

stars, this shock-wave passage can lead to line-splitting at some pulsational phases (e.g. Gillet et al. 1990). Very similar velocity effects were also detected in the small-amplitude pulsating post-AGB star HD 56126 (Lèbre et al. 1996; Barthès et al. 2000; Fokin et al. 2001), and were again attributed to the passage of a shock wave. Note that the UVES spectra were taken just before minimum light, i.e. the phase at which a new shock wave is expected to develop (Gillet et al. 1989). The adoption of planeparallel, LTE Kurucz models can thus only be a first approximation of the real photosphere.

\section{Results and discussion}

The final abundances can be found in Table 2. The first column of this table gives the actual ion; the second column gives the number of lines used; the third one is the mean equivalent width; the fourth column gives the absolute abundances derived $\log \epsilon=\log (N(\mathrm{el}) / N(\mathrm{H}))+12 ; \sigma_{\mathrm{ltl}}$, the fifth column, is the lineto-line scatter; the sixth column gives the abundance relative to the sun $[\mathrm{el} / \mathrm{H}]$. For the references of the solar abundances (seventh column) needed to calculate the $[\mathrm{el} / \mathrm{H}]$ values: see Reyniers et al. (2006). The dust condensation temperatures (last column) are taken from Lodders (2003).

The abundances are also graphically presented in Fig. 5, where the abundance relative to solar $[\mathrm{el} / \mathrm{H}]$ is plotted against condensation temperature. The clear anti-correlation as seen in Fig. 5 is undoubtfully recognised as a depletion pattern: the slightly subsolar abundances of $\mathrm{Zn}$ and $\mathrm{S}$ are expected for a field LMC-star, while iron is depleted by more than a factor of 300. The CNO elements have low condensation temperatures and are typically expected not to be depleted. The low carbon abundance of $[\mathrm{C} / \mathrm{H}]=-0.6$ makes clear that MACHO 82.8405.15 has not experienced a 3rd dredge-up. One remarkable result is the detection of two forbidden [O I] lines at $6300.23 \AA$ and $6363.88 \AA$, both in absorption. The abundances derived from these lines are much higher than the abundances derived from the O I triplet at $6155 \AA$ (see Table 2). The [O I] lines are known not to be very sensitive to non-LTE effects, so this large abundance difference is not expected. The two oxygen abundances can be brought in agreement if one adopts a slightly lower temperature $(5750 \mathrm{~K})$ combined with a very low gravity of $\log g=-0.4$. Such a low gravity is, however, not compatible with the luminosity. The 
Table 2. Abundance results for MACHO 82.8405.15.

\begin{tabular}{|c|c|c|c|c|c|c|c|}
\hline \multicolumn{8}{|c|}{$\begin{aligned} T_{\text {eff }} & =6000 \mathrm{~K} \\
\log g & =0.5(\mathrm{cgs}) \\
\xi_{\mathrm{t}} & =3.5 \mathrm{~km} \mathrm{~s}^{-1} \\
{[\mathrm{M} / \mathrm{H}] } & =-2.5\end{aligned}$} \\
\hline Ion & $N$ & $\overline{W_{\lambda}}$ & $\log \epsilon$ & $\sigma_{\mathrm{ltl}}$ & {$[\mathrm{el} / \mathrm{H}]$} & sun & $T_{\text {cond }}$ \\
\hline$\overline{C I}$ & 13 & 63 & 8.00 & 0.13 & -0.57 & 8.57 & 40 \\
\hline $\mathrm{NI}_{\mathrm{I}}$ & 9 & 46 & 8.04 & 0.16 & 0.05 & 7.99 & 123 \\
\hline OI & 3 & 26 & 8.53 & 0.10 & -0.33 & 8.86 & \\
\hline [O I] & 2 & 102 & 9.16 & 0.08 & 0.30 & 8.86 & \\
\hline $\mathrm{NaI}$ & 4 & 52 & 5.80 & 0.14 & -0.53 & 6.33 & 953 \\
\hline $\mathrm{Mg}_{\mathrm{I}}$ & 2 & 38 & 5.29 & 0.01 & -2.25 & 7.54 & 1327 \\
\hline S I & 3 & 37 & 6.99 & 0.02 & -0.34 & 7.33 & 655 \\
\hline Ca I & 3 & 25 & 4.24 & 0.15 & -2.12 & 6.36 & 1505 \\
\hline Sc II & 1 & 17 & 0.12 & & -3.05 & 3.17 & 1647 \\
\hline Ti II & 4 & 22 & 2.18 & 0.26 & -2.84 & 5.02 & 1573 \\
\hline CrI & 2 & 49 & 3.26 & 0.04 & -2.41 & 5.67 & 1291 \\
\hline CrII & 3 & 39 & 3.40 & 0.07 & -2.27 & 5.67 & 1291 \\
\hline Mn I & 3 & 28 & 3.60 & 0.13 & -1.79 & 5.39 & 1150 \\
\hline $\mathrm{Fe} \mathrm{I}$ & 35 & 30 & 5.08 & 0.25 & -2.43 & 7.51 & 1328 \\
\hline Fe II & 7 & 52 & 4.87 & 0.19 & -2.64 & 7.51 & 1328 \\
\hline Zn I & 4 & 87 & 4.26 & 0.06 & -0.34 & 4.60 & 723 \\
\hline Y II & 1 & 24 & -0.26 & & -2.50 & 2.24 & 1647 \\
\hline Ba II & 3 & 105 & 0.13 & 0.15 & -2.00 & 2.13 & 1447 \\
\hline
\end{tabular}

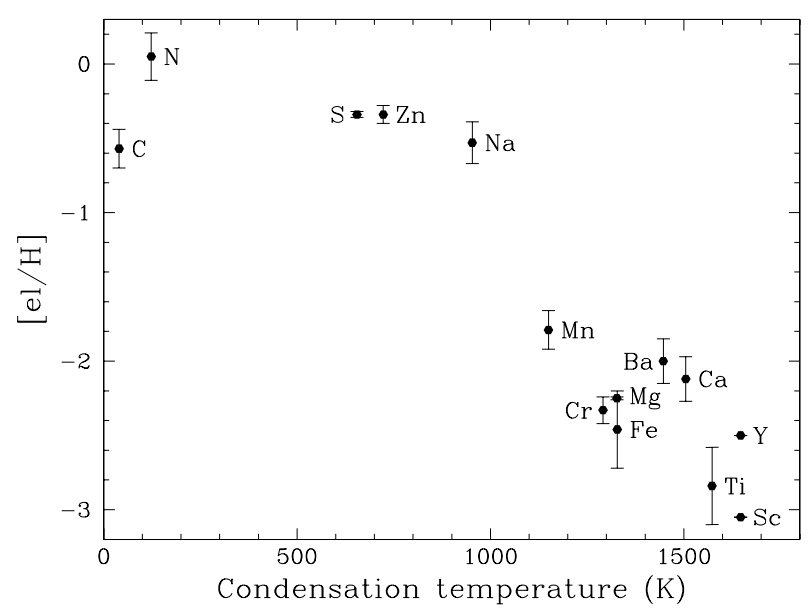

Fig. 5. The anti-correlation between the abundance of an element $([\mathrm{el} / \mathrm{H}])$ and its condensation temperature. This indicates that a very efficient depletion process has taken place in MACHO 82.8405.15. The errorbar is the line-to-line scatter listed in Table 2.

abundances of the s-process elements $\mathrm{Y}$ and $\mathrm{Ba}$ show an expected behaviour and are probably not intrinsically enhanced.

\section{Conclusion}

In this letter, we have found evidence that the photospheric depletion phenomenon, which is frequently observed in field RV Tauri stars, also occurs in the extragalactic members of this pulsation class. The effect on the observed metallicity of MACHO 82.8405 .15 is very significant $([\mathrm{Fe} / \mathrm{H}]=-2.6)$, while the initial iron content was probably only slightly subsolar, as expected in the LMC. While the degree of depletion of MACHO 82.8405.15 is beyond any doubt, the analysis itself can be improved by adopting a more realistic model atmosphere that accounts for the observed dynamics and the non-LTE effects that we encountered in our analysis. Additional spectra at different pulsation phases may reveal interesting results, both those from the LTE approach of a dynamic photosphere and from the abundance determination of lines and species that become accessible at different temperatures.

Although detailed scenario for the depletion process is still lacking, there is growing evidence (Van Winckel 2003; De Ruyter et al. 2006) that binarity plays a key role in the creation of a stable dusty circumstellar environment that is probably needed for the process to occur. High-resolution infrared data of MACHO 82.8405.15 would yield invaluable information on the physical conditions and mineralogy of the circumstellar environment.

Our first extragalactic detection of a depleted photosphere in a luminous star shows that the depletion process may be rather common elsewhere. MACHO 82.8405.15 is a member of a larger sample of luminous LMC RV Tauri objects. Our recent analysis of one of the other objects (MACHO 47.2496.8, Reyniers et al. 2006) shows, however, a very different chemical result with a very strong s-process-enhanced photosphere in an intrinsically metal-poor environment of $[\mathrm{Fe} / \mathrm{H}]=-1.4$. Clearly the LMC RV Tauri stars are also very diverse chemically. A global study of these objects will enable us to better constrain both the depletion process itself, as well as the true evolutionary status of RV Tauri stars.

Acknowledgements. It is a pleasure to thank Clio Gielen for the construction of the SED, Sophie Saesen for the Euler observations, the anonymous referee for the quick and constructive report, and the Geneva staff for observation time on the Euler telescope. MR acknowledges financial support from the Fund for Scientific Research - Flanders (Belgium).

\section{References}

Alcock, C., Allsman, R. A., Alves, D. R., et al. 1998, AJ, 115, 1921 Barthès, D., Lèbre, A., Gillet, D., \& Mauron, N. 2000, A\&A, 359, 168 De Ruyter, S., Van Winckel, H., Maas, T., et al. 2006, A\&A, 448, 641 Deroo, P., Reyniers, M., Van Winckel, H., Goriely, S., \& Siess, L. 2005, A\&A, 438, 987

Fokin, A. B., Lèbre, A., Le Coroller, H., \& Gillet, D. 2001, A\&A, 378, 546 Gillet, D., Duquennoy, A., Bouchet, P., \& Gouiffes, C. 1989, A\&A, 215, 316 Gillet, D., Burki, G., \& Duquennoy, A. 1990, A\&A, 237, 159

Giridhar, S., Lambert, D. L., \& Gonzalez, G. 2000, ApJ, 531, 521

Giridhar, S., Lambert, D. L., Reddy, B. E., Gonzalez, G., \& Yong, D. 2005, ApJ, 627,432

Jura, M. 1986, ApJ, 309, 732

Lèbre, A., Mauron, N., Gillet, D., \& Barthès, D. 1996, A\&A, 310, 923

Lloyd Evans, T., \& Pollard, K. R. 2004, in ASP Conf. Ser., 310, IAU Colloq. 193, Variable Stars in the Local Group, ed. D. W. Kurtz, \& K. R. Pollard, 344 Lodders, K. 2003, ApJ, 591, 1220

Maas, T., Van Winckel, H., \& Lloyd Evans, T. 2005, A\&A, 429, 297

Preston, G. W., Krzeminski, W., Smak, J., \& Williams, J. A. 1963, ApJ, 137, 401 Reyniers, M., Abia, C., Van Winckel, H., et al. 2006

[arXiv:astro-ph/0610240]

Seares, F. H., \& Haynes, E. S. 1908, Laws Observatory Bulletin, University of Missouri, 14, 215

Shchukina, N. G., Bueno, J. T., \& Asplund, M. 2005, ApJ, 618, 939

Thévenin, F. 1989, A\&AS, 77, 137

Thévenin, F. 1990, A\&AS, 82, 179

Van Winckel, H. 2003, ARA\&A, 41, 391

Van Winckel, H., \& Reyniers, M. 2000, A\&A, 354, 135

Van Winckel, H., Waelkens, C., Waters, L. B. F. M., et al. 1998, A\&A, 336, L17 Waters, L. B. F. M., Trams, N. R., \& Waelkens, C. 1992, A\&A, 262, L37 